Voix et Images

volxetimages

\title{
Être écrivaine et " reine du foyer " dans les années 1920 et 1930
}

Les journaux intimes de Michelle Le Normand

Being a Writer and "Queen of the hearth" in the 1920's and

1930's

The Private Journals of Michelle Le Normand

Ser escritora y "reina del hogar" en los años 1920 y 1930

Los diarios íntimos de Michelle Le Normand.

\section{Patricia Smart}

Volume 39, numéro 2 (116), hiver 2014

Voix de femmes des années 1930

URI : https://id.erudit.org/iderudit/1025187ar

DOI : https://doi.org/10.7202/1025187ar

Aller au sommaire du numéro

Éditeur(s)

Université du Québec à Montréal

ISSN

0318-9201 (imprimé)

1705-933X (numérique)

Découvrir la revue

Citer cet article

Smart, P. (2014). Être écrivaine et « reine du foyer » dans les années 1920 et 1930 : les journaux intimes de Michelle Le Normand. Voix et Images, 39(2),

43-56. https://doi.org/10.7202/1025187ar
Résumé de l'article

Dans le Québec des années 1920 et 1930, où la glorification de la femme comme " reine du foyer » était à son comble, les pressions sur les femmes pour se marier et se consacrer à la vocation maternelle étaient énormes, et la difficulté de s'imposer en tant qu'écrivaine demeurait considérable. Le journal intime de la romancière Michelle Le Normand, l'épouse du romancier Léo-Paul Desrosiers et la mère de trois enfants, est un témoignage précieux sur ce conflit, tel que vécu par une femme qui a trouvé dans les idées conservatrices de son temps à la fois un soutien et une entrave à sa liberté de penser et d'écrire. 


\section{ÊTRE ÉCRIVAINE ET “REINE DU FOYER " DANS LES ANNÉES 1920 ET 1930}

Les journaux intimes de Michelle Le Normand

$+++$

PATRICIA SMART

Université Carleton

Pour les femmes qui aspirent à écrire, réconcilier l'activité scripturale avec les responsabilités du mariage et de la maternité est un problème sempiternel. On oublie parfois que les trois plus grandes écrivaines de la littérature québécoise d'avant la Révolution tranquille - Laure Conan, Gabrielle Roy et Anne Hébert - étaient des femmes non seulement sans enfants, mais célibataires dans le cas de deux d'entre elles, Laure Conan et Anne Hébert. Dans le Québec des années 1920 et 1930, où la glorification de la femme comme «reine du foyer" battait son plein et où le vote provincial pour les femmes (obtenu en 1940) était encore un rêve lointain, les pressions sur les femmes pour se marier et se consacrer à ce qu'on présentait comme leur vocation maternelle étaient énormes, et le défi de s'imposer en tant qu'écrivaine se révélait d'autant plus considérable. Le journal intime de la romancière Michelle Le Normand ${ }^{1}$ offre un témoignage précieux sur ce conflit tel que vécu par une écrivaine, mariée et mère de trois enfants, qui a joui d'un certain renom à son époque. Par ailleurs, le fait que Le Normand était l'épouse d'un romancier connu et respecté, Léo-Paul Desrosiers, situe les révélations de son journal dans le cadre d'une littérature critique, souvent d'inspiration féministe, qui porte sur les enjeux de la créativité dans les couples d'artistes ou d'écrivains ${ }^{2}$. Tandis que dans certains de ces couples la vie en commun semble avoir libéré les élans créateurs de chacun des deux partenaires ${ }^{3}$, il arrive plus souvent que la femme, sous l'emprise des pressions sociales, donne la priorité à la carrière de son mari, ne réalisant qu'en partie son propre talent créateur. Aucune étude de

$$
+++
$$

1 En 1909, à l'âge de seize ans, Michelle Le Normand (1893-1964) commence à tenir un journal, pratique à laquelle elle restera fidèle toute sa vie. Vingt cahiers contenant ses journaux rédigés à la main, allant de 1909 à 1911, et ensuite de 1918 jusqu'à son décès en 1964, sont conservés dans le fonds Le Normand-Desrosiers (026/002/054-026/004/005, Montréal, Bibliothèque et Archives nationales du Québec). Désormais, les références aux journaux seront indiquées par la date des entrées, et placées entre parenthèses dans le texte. 2 Voir par exemple Phyllis Rose, Mariages victoriens, traduit de l'anglais par Doris Febvre, Paris, Albin Michel, 1988, 324 p. ; et Whitney Chadwick et Isabelle de Courtivron (dir.), Significant Others. Creativity and Intimate Partnership, New York/Londres, Thames and Hudson, 1995, 256 p. 3 Les exemples les plus réussis de couples artistiques se trouvent dans le domaine des arts visuels : Sonia et Robert Delaunay, Georgia O'Keeffe et Alfred Stieglitz, Frida Kahlo et Diego Rivera, par exemple. 
l'œuvre publiée de Le Normand n'existe, mais il est clair d'après les révélations de son journal que le patriotisme conservateur auquel les Desrosiers adhéraient avec passion, et dans lequel ils cherchaient leur inspiration littéraire, n'était pas propre à nourrir le besoin profond d'écrire, et de s'imposer comme écrivaine, qu'elle évoque si souvent et avec tant de regrets. Comme nous le verrons, d'autres sujets, plus proches de sa vie de femme, l'attiraient, mais elle n'est jamais parvenue à les incarner dans un roman. C'est dans les pages mêmes de son journal que Le Normand trouvera en fin de compte son expression la plus éloquente.

Et pourtant, Le Normand était loin du stéréotype de la femme effacée. Dans une étude de son journal et de sa correspondance, Michel Lacroix montre qu'elle était une femme d'affaires extrêmement efficace, pour qui «les livres et les prix sont des produits comme les autres, soumis aux lois de l'offre et de la demande. Vendre des livres (ou un vote dans un jury) permet d'acheter une auto ou un voyage en France ${ }^{4} »$. À une époque où beaucoup d'écrivains (y compris Le Normand et son mari, ainsi que leurs amis Alfred Lozeau et Lionel Groulx) publiaient à compte d'auteur, Le Normand consacrait un temps et une énergie considérables à la promotion de ses livres et de ceux de son mari auprès des institutions religieuses et éducatives, tout autant que chez des éditeurs en France. Bien que l'image de Le Normand qui se dégage de l'article de Lacroix soit surtout celle de la commerçante-négociatrice, l'auteur note en passant deux «source[s] de tiraillements mettant à mal l'image bien lisse de la femme d'affaires » : le « désir vif mais étrangement inavouable de devenir un véritable écrivain» et «les enjeux propres à la place de la femme dans la famille comme dans le monde littéraire ${ }^{5}$ ». À l'appui, il cite des phrases d'une lettre adressée à Le Normand par Claude-Henri Grignon en 1938: « Je veux la femme à sa place, dans son rôle. Les arts ne lui sont pas interdits. Elle a le droit de s'en occuper à la condition que son mari ne manque de rien. Le mari doit passer avant tout ${ }^{6}$.»

Le présent article explorera la tension entre le désir d'être écrivaine et le devoir d'être une bonne épouse et mère chez Le Normand, en la situant dans le contexte d'un mariage d'écrivains parfois extrêmement tendu et d'une société catholique et conservatrice où les droits des femmes étaient bafoués et le divorce, interdit. Lire ce journal, qui couvre les quelque quarante ans séparant le mariage de Le Normand de sa mort en 1964, est une expérience attristante, au cours de laquelle on a souvent le sentiment de faire intrusion dans un domaine qui devrait peut-être rester privé, car la diariste y note les fréquents moments de désespoir dus à une situation maritale intenable, dans laquelle elle est emprisonnée par les attitudes rigides de son époque et par sa propre vision du monde conservatrice. S'il existe une justification pour une telle intrusion dans une vie privée, elle se trouve dans le courage, la vulnérabilité et la joie de vivre malgré tout qui se dégagent de ce journal.

4 Michel Lacroix, " "Mon petit commerce". Michelle Le Normand, femme de lettres et femme d'affaires», Chantal Savoie (dir.), Histoire littéraire des femmes. Cas et enjeux, Québec, Nota bene, coll. «Séminaires», 2010, p. 175. 5 Ibid., p. 182, 184. 6 Lettre du 22 juin 1938 (MSS026/009/010, fonds Le Normand-Desrosiers), citée dans Michel Lacroix, «"Mon petit commerce”», p. 185. 
Le Normand (de son vrai nom Marie-Antoinette Tardif) a publié neuf livres ${ }^{7}$ entre 1916 et 1964: Autour de la maison, un recueil de réminiscences nostalgiques inspiré peut-être par le succès qu'a connu Chez nous ${ }^{8}$ d'Adjutor Rivard l'année précédente; Couleur du temps, un recueil des billets publiés dans Le Devoir entre 1915 et 1918; trois romans (Le nom dans le bronze, La plus belle chose du monde et La montagne d'hiver); deux recueils de nouvelles (La maison aux phlox et Enthousiasme); un livre de récits (Dans la toile d'araignée); et une biographie (Marie-Célina Plourde, veuve de Joseph-Onias Thériault: sœur Sainte-Jeanne-de-Chantal, des Servantes de Jésus-Marie, 1879-1938). En plus de la chronique qu'elle a rédigée pour Le Devoir de façon intermittente entre 1915 et 1925, Le Normand a donné des articles et des recensions de livres à plusieurs publications durant la décennie 1930, dont Le Canada français, L'Action catholique, Le Droit, Le Devoir et La Revue dominicaine.

L'absence de titres pour la décennie 1920 dans la bibliographie de Le Normand s'explique facilement: pendant ces années, elle se marie et donne naissance à trois enfants. En juin 1922, elle épouse Léo-Paul Desrosiers, le futur auteur de romans historiques et patriotiques, notamment Nord-Sud et Les engagés du grand portage ${ }^{9}$, qui figurent parmi les classiques de la littérature québécoise. Selon Julia Richer, une grande amie des Desrosiers, la complémentarité du couple était remarquable :

\begin{abstract}
Jamais deux êtres aussi dissemblables ne se complétèrent aussi bien. Autant LéoPaul Desrosiers était sérieux, méditatif, silencieux; autant Michelle, sa femme, était vive, primesautière, enthousiaste. Mais ils avaient en commun l'amour de la littérature et un irrépressible besoin d'écrire. Du jour où le sourire de Michelle Le Normand vint combler la solitude de Desrosiers, les jeux furent faits. Appuyé dorénavant par une compagne qui plaçait avant toutes choses le travail intellectuel, il put, en toute sécurité, commencer une œuvre qui, par alternance, s'élaborera sur deux plans : l'histoire et le roman ${ }^{10}$.
\end{abstract}

Derrière cette image projetée par le couple Desrosiers, on soupçonne les tensions possibles entre deux êtres aussi dissemblables, l'un "sérieux, méditatif, silencieux» et l'autre pleine de joie de vivre; l'un considéré comme un grand écrivain et l'autre comme la compagne qui appuie son mari et lui assure la sécurité nécessaire à l'édification de son œuvre. Michelle Le Normand était en effet dévouée à son mari et convaincue de la grandeur de son œuvre; c'était une collaboratrice essentielle qui

$$
+++
$$

7 Michelle Le Normand, Autour de la maison, Montréal, Éditions du Devoir, 1916, 155 p.; Couleur du temps, Montréal, Éditions du Devoir, 1919, 142 p. ; Le nom dans le bronze, Montréal, Éditions du Devoir, 1933, 163 p.; La plus belle chose du monde, Montréal, Éditions du Devoir, 1937, 249 p. ; La montagne d'hiver, Montréal, Fides, coll. «La gerbe d'or», 1961, 158 p.; La maison aux phlox, Ottawa, [s. ê.], 1941, 212 p. ; Enthousiasme, Montréal, Éditions du Devoir, 1947, 222 p. ; Dans la toile d'araignée, Montréal, Éditions Jeunesse, 1949, 183 p. ; Marie-Célina Plourde, veuve de Joseph-Onias Thériault: sœur Sainte-Jeanne-de-Chantal, des Servantes de Jésus-Marie, 18791938, Montréal, [s. é.], 1942, 150 p. 8 Adjutor Rivard, Chez nous, Québec, L'Action sociale catholique, 1914, 145 p. 9 Léo-Paul Desrosiers, Nord-Sud, Montréal, Éditions du Devoir, 1931, 199 p. ; Les engagés du grand portage, Paris, Gallimard, 1938, 209 p. 10 Julia Richer, Léo-Paul Desrosiers, Montréal, Fides, coll. «Écrivains canadiens d'aujourd'hui», 1966, p. 19-20. 
corrigeait et recopiait les manuscrits de Desrosiers en plus de s'occuper de ses relations avec les éditeurs et même de la vente de ses livres. Mais son journal révèle le prix qu' elle a payé et la réalité derrière la façade de la complémentarité parfaite du couple : les ravages exercés sur son propre sentiment de soi par la morosité constante de son époux, et sa lutte de tous les instants pour continuer à écrire elle-même malgré les obstacles posés par ses responsabilités d'épouse et de mère.

La perspective inacceptable d'une carrière littéraire qui aurait exclu le mariage et la maternité avait déjà mené Le Normand à rompre avec son mentor Alfred Lozeau en 1919, étant donné que celui-ci, follement amoureux d'elle, était infirme et jugé inapte aux liens conjugaux: "[Je ne veux] plus regarder ma vie comme une longue carrière littéraire où le cœur se serait occupé de consoler un malade, mais la regarder, avec dans le fond, un mariage probable, et la maternité, et des sentiments et des actions canadiennes », confie-t-elle à son journal le 21 avril 1919. À vingt-sept ans, elle a dû voir en l'amour de Desrosiers, un homme qui partageait ses interêts littéraires, patriotiques et religieux, une bouée de sauvetage. Dès les premières lettres de celui-ci, elle remarque cependant qu'il est mélancolique, hypersensible, «un jeune homme triste et pas très heureux " (14 décembre 1920). Desrosiers, pour sa part, est très épris d'elle, et en décembre 1921, pendant un long séjour à Paris, elle décide d'accepter sa demande en mariage. Se déclarant éperdument amoureuse, elle abandonne l'écriture pendant plusieurs mois, notant: «Je travaille peu ma littérature et beaucoup mon trousseau.» (22 mars 1922) Au cours des années suivantes, ce thème de «ma littérature » reviendra souvent sous sa plume, évoquant presque toujours des sentiments de frustration et de culpabilité provoqués par le peu de temps qu'elle arrive à consacrer à l'écriture.

\section{LES PREMIËRES ANNÉES DE MARIAGE (CAHIER VII, JUIN 1922-OCTOBRE 1928)}

Le Normand commence un nouveau cahier de son journal pendant sa lune de miel avec Desrosiers au Bic, chacun des deux partenaires y inscrivant leur bonheur d'être ensemble. Ce cahier couvrira plus de six ans, indiquant le peu de temps qu'elle trouvera pour y écrire entre les activités de sa vie de jeune épouse et de mère. Le couple s'installe ensuite à Ottawa, où Desrosiers est chroniqueur parlementaire pour Le Devoir; en 1928 il quittera Le Devoir pour devenir rédacteur du feuilleton et des procès-verbaux à la Chambre des communes, poste qu'il occupera jusqu'en 1941. Un premier fils, Louis, naîtra en 1923, et un deuxième, Claude, en 1925. Ce sont des années généralement heureuses, à tel point que, pendant la première année, Le Normand se dit parfois angoissée par son trop parfait bonheur : «Nous sommes tellement, tellement heureux ensemble que parfois je m'arrête à avoir peur rien qu'un peu, pour que l'appréhension me préserve d'autre chose.» (12 mars 1923; Le Normand souligne.) Elle déclare souvent son amour pour Léo-Paul : « Je voudrais toujours qu'il soit parfaitement bien. Un rien m'inquiète. Je l'aime tant» (14 mai 1923); et, le jour de leur premier anniversaire de mariage: «La vie en commun ne nous a ni l'un ni l'autre désappointés. J'en remercie beaucoup le bon Dieu.» (12 juin 1923) En août 1925, elle note qu'ils ont déménagé dans la «maison de [leurs] rêves». 
Et pourtant, les difficultés se font sentir très tôt: frustrations au travail pour Desrosiers, qui iront en s'aggravant quand il sera devenu fonctionnaire, et surtout difficulté d'écrire pour Le Normand. Dès la naissance de son premier fils en avril 1923, elle tient de moins en moins souvent son journal, sauf pour noter le progrès de «bébé». Et puis, en septembre, cet aveu:

Parfois, j'ai de grosses dépressions, à cause de ma littérature. Il me semble que je n'écris plus rien qui vaille, et que je serais si bien, si bien de ne plus écrire. Ce matin - j'ai cet état d'âme. Et je ne vois rien, rien à dire dans mon prochain article. Paul me console, me ravigote - et je sais bien que soudain, je passerai de ce découragement à l'enthousiasme. [...] C'est dur, souvent. Mais on n'a rien sans peine, sans ennui, et je ne devrais pas me laisser abattre pour si peu. (25 septembre 1923)

Une rengaine fréquente au cours de ces années est la difficulté de trouver une bonne (plusieurs sont engagées, qui sont congédiées ou démissionnent peu après). Le peu de temps pour écrire, joint à un manque d'inspiration sans doute relié aux contraintes de son existence de jeune mère de famille, mine peu à peu la confiance de Le Normand, qui n'arrive plus à trouver des sujets pour les billets qu'on attend d'elle au Devoir :

Mon seul souci, c'est mon manque de confiance littéraire, ma stérilité trop répétée! Une fois par semaine, régulièrement, l'article à faire me jette dans la tristesse. Puis, quand il est fini, je me ranime et retrouve ma joie. Paul me gronde. Mon cerveau estil fatigué ? Ou les sujets sont-ils ici un peu rares, avec ma vie unie de tous les jours? (mars 1924)

En août 1925, trois mois après la naissance de son deuxième fils, elle démissionnera du Devoir.

Le journal dépeint de façon exemplaire le tiraillement entre soi et l'autre, entre la vie et l'écriture ressenti par les femmes artistes au cours des générations. Pour être artiste, il faut une certaine dose d'égoïsme, de la discipline, des modèles sur lesquels s'appuyer et, comme disait Virginia Woolf, « une chambre à soi» où se retirer à l'écart du monde: caractéristiques ou conditions qui semblent avoir toutes (ou presque toutes) fait défaut à Michelle Le Normand. Trop sociable peut-être, ou trop amoureuse des plaisirs de la vie, elle émet souvent des doutes par rapport à ce qu'elle appelle «ma littérature », s'accusant de paresse et se culpabilisant quand elle n'a rien produit. Déjà en 1919, elle avait identifié avec précision les enjeux du choix que doivent faire beaucoup de femmes artistes:

Que je suis femme (!) avec mon pauvre cœur qui a besoin d'être sans cesse à quelqu'un et occupé; que je suis femme et que mon imagination est excitée par mon sentiment plus que par ma littérature. C'est égal, je souffre un peu de voir que je ne peux pas assez faire pour ma littérature. Il me semble que je n'avance pas. Je dois faire un article sans faute aujourd'hui. (27 avril 1919) 
Après la naissance de ses deux premiers enfants, elle découvre une nouvelle passion, le tennis, à laquelle elle s'adonne avec son enthousiasme habituel: "C'est exactement une folie. Je jouerais dans la maison si je pouvais. J'ai de grands frissons d'impatience à regarder les courts devant chez nous et à être ici - sans jouer.» (Juillet 1927; Le Normand souligne.) Mais, toujours, la nécessité d'écrire et d'être digne de la réputation dont elle jouit la ramène à sa table de travail : «Il faut compter maintenant bientôt douze ans que j'ai ma petite réputation d'écrivain - et si peu d'œuvres, pour tant d'années. Ce serait si beau d'écrire bien et beaucoup ", écrit-elle, tout en ajoutant: "L'événement - en ces jours - c'est le tennis.» (mai 1927) Revenant quelques mois plus tard à ce même sentiment d'être indigne de son statut d'écrivaine, elle se reproche de n'avoir «rien donné au monde», comptant pour peu le fait d'avoir donné naissance à deux enfants: «Saisie d'une grande confusion en songeant que j'écris depuis 12 ans, que ma gloire rapetisse au lieu de grandir - et que je n'ai encore rien donné au monde.» (29 août 1927)

La maternité semble avoir été difficile à assumer pour Le Normand, qui se lamente parfois de devoir "garder» les enfants quand il n'y a pas de bonne, et qui se rebiffe devant le premier devoir d'une «reine du foyer», celui de se sacrifier pour d'autres: «Je suis égoïste. J'ai été gâtée, petite, et toute ma vie je m'en ressentirai. Je n'aime pas à être sacrifiée, je n'aime pas à me dévouer - ce qui fait que les moindres sacrifices me coûtent plus cher qu'ils ne coûtent à d'autres", écrit-elle le 21 février 1928 (Le Normand souligne). Après une dispute à propos des enfants, à la suite de laquelle son mari boude pendant dix jours, lui reprochant de ne pas être une «bonne mère », elle éclate : «Est-ce ma faute si j'ai été gâtée? si, jusqu'à mon mariage, je n’ai eu qu'à penser à moi - et si j'ai été entourée de gens qui m’ont toujours adulée?» (17-18 juillet 1928)

Les tensions dans le couple augmenteront au cours de ces premières années de mariage. En mars 1927, Le Normand note: «Paul a une tendance au pessimisme prononcée [...] qui m'afflige et qui m'effraye.» Un an plus tard, ce sera la plainte sempiternelle des épouses: «Je trouve Paul trop tranquille, trop uni - l'air d'être trop bien habitué à ma compagnie. Souvent, il ne se souvient pas des choses que je lui ai racontées - et il s'étonne que j'en sois offensée. Je voudrais écrire... continuellement. Cela tuerait mes chimères, mes peines, mes autres exigences.» Et, un peu plus loin: «Au lieu de parler, de projeter, de m'expliquer tout, Paul est muet et taciturne dans toutes les crises. Et il fuit.» (printemps 1928)

\section{ÉCRIRE MALGRÉ TOUT \\ (CAHIERS VIII ET IX, NOVEMBRE 1928-JUIN 1938)}

Pendant la décennie suivante, Le Normand tient un calepin à part (le Cahier VIII) dans lequel elle écrit des ébauches des articles qu'elle publiera, ainsi que des romans Le nom dans le bronze et La plus belle chose du monde. Dans le Cahier IX, elle consigne, de façon intermittente, les événements (notamment la naissance de sa fille Michelle en 1929) et les états émotifs de ses journées. Il s'agit d'un grand cahier épais de trois cent cinquante pages, rédigé de l'âge de trente-cinq à quarante-cinq ans par une 
femme courageuse et exigeante envers elle-même. Pendant cette période, Le Normand mûrit et son écriture embellit: le journal contient quelques belles évocations de paysages d'Ottawa ou de la Gaspésie, où le couple passe plusieurs semaines chaque été, et des descriptions qui évoquent merveilleusement la configuration de sa vie quotidienne. Par la force des choses, elle devient plus réaliste, se débattant constamment au cours de ces années avec deux lourds fardeaux : le marasme maladif de son mari et l'état anormal de sa fille Michelle.

Le journal s'ouvre sur un thème familier - la difficulté d'écrire - , dans un passage qui évoque bien la texture de l'expérience quotidienne et les multiples distractions qui empêchent la diariste de travailler:

\begin{abstract}
Il pleut, il fait sombre, je n'ai rien dans la tête et pas d'entrain. Je pourrais pourtant travailler, puisque mes deux garçons s'amusent assez sagement en bas. Mais il me faudrait de la lumière !... de la lumière au-dehors, de la lumière en dedans. Mon automne est souvent morne. Je prends des résolutions littéraires féroces, puis, le temps, les circonstances, ou ma paresse les anéantissent. (3 novembre 1928)
\end{abstract}

En plus de ses responsabilités à la maison, Le Normand est pleinement engagée dans la vie de la bourgeoisie francophone d'Ottawa. Elle est active dans plusieurs cercles, fait des causeries, écrit parfois des billets et des recensions de livres, entretient des amitiés et reçoit de temps en temps une vingtaine ou une trentaine de personnes à l'heure du thé. Elle se débat souvent pour trouver du temps pour écrire, incapable de renoncer à ses ambitions littéraires: "Essayé deux heures hier de mettre à point le début de mon roman [...]. Raté mon coup [...], découragée [...]. Peut-être n'ai-je pas le souffle qu'il faut pour un long travail. Et pourquoi, tout de même, ce souci de l'œuvre à faire qui ne me quitte jamais ? (19 avril 1929) Pourtant, elle a trois romans en chantier: Le nom dans le bronze, roman nationaliste inspiré par les idées de l'abbé Lionel Groulx, qui paraîtra en $1933^{11}$; un livre dont le titre provisoire est Quatuor ou Les quatre, qui deviendra La plus belle chose du monde, roman d'inspiration autobiographique sur quatre jeunes femmes vivant à Montréal au début de la Première Guerre mondiale; et enfin un autre roman, plus proche de sa vie de mère et d'épouse, auquel elle songe souvent, mais qu'elle n'écrira jamais. Évoquant son manque d'inspiration pour un roman qu'elle essaie de faire démarrer (probablement Le nom dans le bronze), elle admet qu'il ne correspond pas assez à sa propre expérience et se dit que le prochain roman, qui s'appellera Maternité, sera meilleur et plus facile à écrire (26 novembre 1928).

Le nom dans le bronze, avec son intrigue semblable à celle du roman controversé de Groulx, L'appel de la race ${ }^{12}$, raconte l'histoire d'une jeune femme éprise d'un anglophone qui, lors d'un voyage à Québec, découvre l'importance de son héritage culturel et décide de renoncer à sa relation amoureuse. Le Normand semble elle-même mal à

11 Le Normand lisait Barrès et endossait son nationalisme de droite dès le début des années 1920, et surtout, elle et son mari étaient des amis et des disciples de l'abbé Lionel Groulx. 12 Lionel Groulx, L'appel de la race, Montréal, Bibliothèque de l'Action française, 1922, 278 p. 
l'aise avec la teneur idéologique de ses propos, craignant que le patriotisme soit un thème démodé et que son livre soit mal reçu par les critiques (3 mars 1933). Au cours de la rédaction, elle envoie le manuscrit à trois prêtres pour le faire approuver, procédé qui lui semble "une idée baroque», mais auquel elle se plie. Quand un des trois émet des réserves sur le livre, elle s'exclame: "Un abbé connaît-il quelque chose à des romans? Et celui-ci qui est poète en plus!» (24 novembre 1932) Le roman fut très bien accueilli par la critique lors de sa parution. Mais les incertitudes de l'auteure se font sentir dans la dédicace, écrite de sa main, qui se trouve dans l'exemplaire de la collection de la Grande Bibliothèque à Montréal : «À Berthelot Brunet ${ }^{13}$, ce livre que vous allez probablement détester.»

Le Normand et Desrosiers rêvaient de renommée internationale, avec une certaine justification, puisque Nord-Sud de Desrosiers et Autour de la maison de Le Normand avaient été couronnés par l'Académie française en 1932, et que Desrosiers avait reçu une lettre élogieuse de Gaston Gallimard l'invitant à publier chez lui (2 juin 1932). Dès avril 1934, Le Normand commence son roman La plus belle chose du monde, s'en remettant à Dieu (comme elle le fait souvent) pour que son travail réussisse: «Ô Dieu, faites que j'écrive un chef-d'œuvre, faites au moins que ma carrière d'écrivain soit réussie. La réussir, ce serait du patriotisme : frapper un gros coup, être connus à l'étranger Paulo et moi. Ô le beau rêve! Faudra-t-il celui-là aussi le sacrifier?» (été 1935) En août, elle note avec satisfaction que "la plume toute seule se met à courir...», ajoutant: "Ce n'est pas personnellement pour moi que je voudrais la gloire, c'est pour mon pays, pour mes enfants.» (1 ${ }^{\mathrm{er}}$ août 1935) Puis, pendant quatre mois en 1936, elle fait passer un essai de son mari, Le livre des mystères ${ }^{14}$, avant son propre livre, s'occupant des corrections d'épreuves, de la publicité, de la vente et de l'envoi du volume. En mai 1937, apprenant que Grasset refuse de publier le manuscrit qu'elle leur a soumis, elle se résigne au fait qu'elle ne sera pas éditée en France. Elle publiera donc elle-même son manuscrit, par l'entremise du Devoir (10 septembre 1937). Lors de sa parution, La plus belle chose du monde recevra l'approbation unanime des critiques.

Ce sont donc des années productives, pendant lesquelles le couple Le Normand-Desrosiers semble aller de triomphe en triomphe; toutefois, en toile de fond, le journal révèle une atmosphère de crispation constante et même de tragédie, car chaque année de la décennie 1930 leur révèle davantage le grave état d'arriération mentale et physique de leur fille. Entre le mari et la femme, le conflit de tempéraments, au lieu de s'apaiser, s'aggrave. Dans l'intimité de son journal, Le Normand donne libre cours à ses émotions de colère et de désespoir, accusant son mari d'avoir détruit sa joie de vivre en agissant toujours en éteignoir. Le jour de leur septième anniversaire de mariage, elle écrit: «[I]l me semble que [...] Paul sera toujours malade, et j'ai peur de cette vie qui à la longue va me changer. Cette nuit, il me disait: "Tu n'aurais pas dû m'épouser, pauvre Miche!" »(12 juin 1929) Les tensions de Desrosiers dues à son

13 Critique et romancier, Berthelot Brunet était l'auteur de plusieurs livres, dont Les hypocrites (Montréal, Éditions de l'Arbre, 1945, 238 p.) et Histoire de la littérature canadienne-française (Montréal, Éditions de l'Arbre, 1946, 186 p.). 14 Léo-Paul Desrosiers, Le livre des mystères, Montréal, Éditions du Devoir, 1936, 175 p. 
travail à la Chambre des communes s'exacerbent pendant cette décennie, le plongeant dans un état de dépression permanent. Quant à Le Normand, seuls le travail et la reconnaissance de ses livres et de ceux de son mari lui permettent de retrouver sa verve. Le jour du trente-cinquième anniversaire de son mari, elle note: «[Devant] sa tristesse incurable, $[\ldots]$ je travaille. C'est la seule consolation. Et puis, je m'enthousiasme, quand des commandes de livres m'arrivent. Nord-Sud a paru...» (4 novembre 1931) Bien qu'elle sympathise entièrement avec son mari en ce qui concerne ses frustrations à la Chambre des communes, elle se rend compte que ses problèmes sont d'ordre psychologique aussi bien que professionnel: «Il n'est pas sociable. Il n'aime personne, au fond, n'est pas fait pour avoir des amis. [...] Trop seul, trop à méditer et à mijoter.» (21 décembre 1932) Quand le prix David est décerné à Louvigny de Montigny et non pas à son mari en 1932, elle est pleine d'amertume, brûlée par ce qu'elle conçoit comme une injustice, $\mathrm{d}^{\prime}$ autant que «L. de Montigny a maintenant une auto : son jury l'a payée!» (12 novembre 1932) Ses propres humeurs, remarque-t-elle, dépendent étroitement de celles de son mari: «Il ne sait pas vivre, dans le sens de donner un sens à sa vie. Tout vient de là, ses humeurs noires, ses songeries [...]. Quand il est gai, quand il a le sourire facile, je suis tout de suite en pleine félicité » (10 mai 1934); «chaque crise le soulage et [...] lui redonne son équilibre. Mais mon équilibre à moi, chaque fois s'affaiblit» (28 octobre 1937).

La découverte graduelle par Le Normand que sa fille Michelle n'est pas normale est d'une lecture déchirante. Pendant sa première année d'existence, la petite est souriante et douce, mais lente à se développer; parfois, sa mère observe qu'elle est «paresseuse». À dix-neuf mois, elle ne marche toujours pas, et sa mère s'affole, d'autant plus angoissée qu'elle a le sentiment de porter seule le fardeau de la situation: "Quand on s'imagine, en se mariant, qu'on prend un soutien, on se trompe souvent. $\mathrm{Au}$ fond, c'est partout où je vais la femme qui soutient, garde le sang-froid, le courage.» (22 juin 1931) À trois ans et demi, aucun changement: «Ma petite fille ne marche pas. Je ne pense jamais à autre chose et tout le monde me croit si gaie, si heureuse. » (25 mai 1933) Et, quelques mois plus tard : «Sera-t-elle un jour normale? parlant, marchant comme les autres? J'en doute, parfois, et ne me console qu'en pensant qu'elle n'est pas malheureuse, qu'elle est gaie, et choyée, et que nous l'entourons de tendresse et de soins. » (2 octobre 1933) Un an plus tard, après consultation avec plusieurs médecins, elle parle d'une mystérieuse «maladie d'une invisible glande qui fait qu'à cinq ans bientôt, elle ne marche pas seule, et ne dit que "papa" et "maman" " (3 octobre 1934). Quelques jours plus tard, le frère André, envoyé par la belle-sœur de Le Normand, qui est religieuse, viendra voir la petite; visite qui émeut Le Normand et la fait pleurer d'angoisse, parce que désormais elle se résigne au fait que «Dieu ne veut pas un miracle» (14 octobre 1934). En 1935, le diagnostic du médecin est sans merci : « [I]nutile de la traiter... elle ne s'améliorera qu'un peu et mourra avant d'atteindre la puberté.» (22 septembre 1935) Le Normand prie constamment pour sa petite, et, bouleversée par l'idée qu'il faudra la placer en pension, peut-être à SaintJean-de-Dieu, avoue: «Puisque je n'obtiens pas de miracle, je voudrais - moi - que le bon Dieu vienne la chercher. » (15 juin 1936) En 1937, elle se lamente : «Cette petite vie incomplète, végétative, gazouillante et sans joie [...] ne profite à personne, qu'à ma sanctification, ma souffrance.» (28 avril 1937) 
Impuissante à changer la réalité de sa situation, Le Normand se tourne de plus en plus vers la religion, trouvant une certaine consolation - comme tant d'autres femmes de l'époque - dans l'exaltation janséniste de la souffrance et du sacrifice. Devant la morosité de son mari, elle constate: «Rien à faire, qu'à endurer. Mes tentatives [sont] trop souvent rebutées.» (9 mai 1935) L'endurance est évoquée aussi à propos des luttes de son mari : « [Léo-Paul] en a tant eu à endurer - mais Dieu qu'il a été mal bâti pour vivre. Je prie. Je n'ai plus d'autre ressource. Et au lieu d'être aussi douce et patiente, je me fâche - oh! sans cris! - et lui dis ce que je pense. Son esprit soupçonneux et compliqué me désespère.» (8 juillet 1936)

\section{TOUCHER LE FOND... \\ (CAHIER X, 2 AOÛT 1938-1 ${ }^{\text {er }}$ MAI 1944)}

Un changement majeur dans la fortune des Desrosiers surviendra en 1941, lorsque Léo-Paul Desrosiers sera nommé conservateur de la Bibliothèque municipale de Montréal et, l'année suivante, élu à la Société royale du Canada. Le Normand consigne ces événements dans le dixième cahier de son journal, ainsi que le plus grand calme qui s'installe dans la vie du couple après le déménagement à Montréal. Toutefois, la plus grande partie de ce cahier de cent quatre-vingts pages est consacrée aux dernières années à Ottawa, la période la plus difficile de leur vie ensemble. À cette époque, Le Normand travaille à un roman qui ne verra jamais le jour, mais dont le titre, La lampe est éteinte, évoque le désespoir qu'elle ressent. Ce sera un roman, dit-elle, qui tentera de communiquer l'expérience de l'incommunicabilité dans un couple, tout en satirisant la stérilité du milieu de la fonction publique à Ottawa : «Le roman que j'ai dans l'idée serait l'histoire d'un jeune ménage [...] et je voudrais essayer de capter cette mélancolie du moment que nous traversons: quand je n'ai plus jamais l'impression que je suis pour Paulo ce qu'il y a de meilleur au monde, quand j'ai plutôt la peur constante de déplaire, d'être critiquée. » (23 octobre 1938) Durant leurs vacances en Gaspésie à l'été 1939, elle parle de son roman qui avance, mais «qui ne sera pas publiable tant que Paulo sera au service civil» (7 août 1939), ajoutant, quelques mois plus tard: «Mais cela ne rend pas le livre inoffensif. Il restera dangereux à publier pour des gens dans le service public. [...] [M]oi, personnellement, je serais pour l'audace, quel qu'en soit ensuite le prix. Et un succès de scandale ne me déplairait pas.» (29 novembre 1939) En 1943, elle y travaille encore, faisant le portrait de l'ancien patron détesté par son mari, le sénateur Rodolphe Lemieux ${ }^{15}$, «maintenant mort et enterré [bien que] son portrait continue à sourire dans les couloirs de la Chambre des communes » (10 août 1943).

Ce sont toutefois des années de succès littéraire pour elle. En février 1939, son roman La plus belle chose du monde paraît en France; elle fait mention dans son

15 Rodolphe Lemieux C.P. (1866-1937), député libéral et ministre influent dans les cabinets de Wilfrid Laurier et de William Lyon Mackenzie King, était président de la Chambre des communes et membre du conseil privé pendant les années où Desrosiers travaillait à la Chambre. En 1930, il fut nommé au Sénat. 
journal d'une lettre et du compte rendu d'une conférence donnée par un certain Monsieur Bruneau sur le roman moderne, dans laquelle il aurait dit: "En 1939, c'est Madame de Sévigné qui est au programme de licence en Sorbonne; en 2039, ce sera peut-être Madame Michelle Le Normand. (11 septembre 1939) Son horaire de travail est chargé : en février 1941, elle publie un recueil de billets et de nouvelles intitulé $L a$ maison aux phlox; elle travaille à sa biographie de sœur Sainte-Jeanne-de-Chantal ainsi qu'à des billets et des recensions de livres pour plusieurs publications; et elle continue de corriger les épreuves et de s'occuper des ventes des livres de son mari. Le 22 octobre 1938, elle note: "Paulo me fait sans cesse des reproches parce que je ne travaille pas. Mais il oublie peut-être tous les jours où je n'écris pas pour moimême parce que je réponds aux lettres auxquelles il devrait répondre, où que je m'occupe de ses propres livres, de sa publicité.»

En ce qui concerne son mariage, les années 1938-1940 constituent la période la plus noire de sa vie. Elle se questionne souvent sur le bonheur et l'amour dans le mariage, trouvant toujours les mêmes réponses: prier, endurer, se taire, se résigner au malheur en espérant gagner l'Éternité: «Je me disais : ça ne me fait plus rien tout ça, je serai heureuse quand je serai morte, parce que j'aurai enduré chrétiennement tout ça. Alors, c'est tout ce qui importe. Endurer avec le plus de patience, le plus de douceur possible. Endurer. Endurer que tout ce qu'on fait déplaise ou sans raison attriste...» (18 novembre 1938) Les «Fiat, fiat, fiat» et les «À Dieu va» ponctuent son journal pour clore les sujets difficiles, et cependant la résignation ne lui est pas facile : «Sur quelle tendresse compter? Sur quoi compter, d'ailleurs, en ce monde? Et aimer ses souffrances, les recevoir avec une joie mystique, ce n'est pas facile. » (17 mai 1939) Une phrase d'un roman qu'elle adore, Sparkenbroke ${ }^{16}$ de Charles Morgan, l'amène à s'interroger sur l'incommunicabilité entre les êtres:

\footnotetext{
[Je me demandais] comment les humains avec toute leur puissance de langage arrivent si mal à communiquer entre eux [...]. Et partie de cela, je songeais avec tristesse à tout ce que Paulo ne me dit plus, et à ce que je ne lui dis plus sans qu'il cesse, pourtant, d'être ma constante préoccupation. Je n'arrive pas à lui donner le bonheur. Personne n'arrive à donner le bonheur à personne. Et pourtant, malgré l'égoïsme qui fait le fond de ma nature, c'est pour son succès, ses réussites, que je prie constamment. Il est vrai que cela revient à moi au fond. Quand il est content, je peux être heureuse à mon goût. (23 octobre 1938)
}

Parfois, on la sent proche d'une révolte féministe, mais ses croyances conservatrices l'empêchent de suivre sa pensée jusqu'au bout: «Mais le bonheur conjugal, est-ce que ça existe quelque part? Un homme juste est-ce que ça existe aussi ? Pourquoi subissons-nous toutes les mauvaises humeurs des hommes, comme si c'était nous les responsables? [...] [S]ans doute à cause d'Ėve. Tout est venu d'elle.» (6-7 novembre 1938) Et plus loin: 
Je me suis illusionnée longtemps, j'ai tenu à croire au bonheur - mais au fond, déjà depuis bien des années, ce bonheur n'a existé que par intermittence. Paulo ne sera jamais un content. Il est plus seul que moi, parce que si peu sociable. Il ne veut voir personne. Il ne répond même pas au téléphone. [...] Paul n'en guérit pas. C'est comme une gangrène. ( 9 décembre 1938)

En septembre 1939, assoiffée de tendresse, elle fait un rêve étrange sur Albert Lozeau, mort depuis seize ans: "Pourtant je ne désire pas le retour de l'amour», écrit-elle au réveil ; «Je suis durcie. [...] Je ne crois plus au bonheur humain.» (30 septembre 1939) En juillet 1940, en vacances en Gaspésie avec les enfants, elle reçoit une lettre de Desrosiers proposant une solution à leur mésentente : elle irait vivre à Montréal tandis que lui resterait à Ottawa. Démolie, elle passe la journée à prier Dieu pour qu'Il « donne gratuitement le ciel à sa pauvre, pauvre petite fille», avant de conclure: «Je ne sens plus aucun sentiment de P. envers moi [...], que blâme, hostilité, irritation. Il m'a classée $[\ldots]$ au nombre de ses ennemis. » (25 juillet 1940)

Troublantes à lire, de telles révélations sur les moments les plus intimes d'un mariage éveillent le désir de connaître le point de vue de l'autre : possibilité qui peut se réaliser en lisant une correspondance, mais qui n'est pas offerte au lecteur d'un journal intime. Quoi qu'il en soit, les tensions diminuent après le déménagement du couple à Montréal en 1941, et après la difficile décision de placer leur fille (maintenant âgée de onze ans) dans une institution: "Allons-nous nous habituer? Finirons-nous d'être brûlés, au fond de nous, par cette lancinante pitié! Ma petite fille! Ma petite fille! » écrit Le Normand (20 août 1941).

\section{UNE FEMME DE SON TEMPS}

Loin de s'opposer aux idées conservatrices de son époque, Le Normand semble y avoir trouvé une certitude et un sentiment de sécurité qui l'ont soutenue dans ses moments de détresse et ont affermi sa conviction d'avoir en quelque sorte une "mission" à accomplir dans la vie et par l'écriture. Les centaines de livres qu'elle a lus au cours des années, soigneusement notés dans son journal, révèlent la rigidité et l'autocensure imposées à ses goûts littéraires par l'orthodoxie catholique. À côté d'une multitude d'auteurs médiocres, de best-sellers anglais et américains et de livres de spiritualité, on trouve des mentions d'auteurs qu'elle aime: Marcel Proust, Virginia Woolf, Katherine Mansfield, Edith Wharton, Thomas Mann - et Louis Hémon, dont elle aimerait avoir écrit le Maria Chapdelaine (14 octobre 1932). Mais en général la littérature française l'enrage par sa franchise en matière de sexualité et de sensualité, inspirant des explosions colorées à travers les décennies. Après la lecture d'un roman français qu'elle aime (Le grand silence blanc ${ }^{17}$ de Louis-Frédéric Rouquette), elle écrit: «Presque pas un mot d'amour. Quel record pour un Français!» (novembre 1928) Les

17 Le grand silence blanc (avec des illustrations de Clarence Gagnon, Paris, Mornay, 1928 [1921], 237 p.) est un récit d'aventures situé dans le Grand Nord. 
romans français modernes lui semblent «si vides, si peu vrais, tellement peu émouvants» (14 octobre 1932), Les Thibault de Roger Martin du Gard ${ }^{18}$ lui inspirant par exemple la réflexion suivante:

\begin{abstract}
Comme dans tous les romans français, l'amour défendu occupe une place bien grande, et Dieu, que ça m'ennuie. Achevé hier encore Night and Day de Virginia Woolf - où, au contraire, avec des sentiments purement humains mais propres, et avec des gens honnêtes qui ne pensent pas à ça, tout le temps, on nous tient sous le charme 600 pages! (5 janvier 1932)
\end{abstract}

Lisant L'immoraliste ${ }^{19}$ de Gide, elle se demande : «Comment aime-t-on tellement cet auteur, pourquoi sa réputation mondiale?» (été 1933) Le romancier Joseph Malègue, par contre, qu'elle appelle «un Proust catholique», l'enthousiasme: « [C'est] le plus beau roman français que j'ai lu dans ma vie [...], on peut être humain sans jouer dans la boue, sans étaler la vie sexuelle, le mal.» (25 novembre 1934) Elle trouve Les jeunes filles ${ }^{20} \mathrm{~d}$ 'Henry de Montherlant «décadent» (hiver 1937); elle déteste le Journal d'un curé de campagne ${ }^{21}$ de Georges Bernanos (été 1938) et La pharisienne ${ }^{22}$ de François Mauriac (hiver 1942); elle aime Colette, tout en disant: «Que cette femme malhonnête par nature écrit bien. » (automne 1939) Ses goûts ne changeront pas; à la fin des années 1950, elle sera encore indignée par les auteurs de choix en France (Jean-Paul Sartre, Simone de Beauvoir, Albert Camus et André Malraux): «Qu'est-ce que nos bonnes gens ont à apprendre de ces auteurs condamnés par l'Église!» (5 mars 1959) En 1964, elle est ravie d'entendre le père Émile Legault dire à la radio que Sartre est dépassé.

En un sens très réel, c'est ce même conservatisme qui a assuré la réputation de Le Normand et les ventes de ses livres, les communautés religieuses et les écoles ayant constitué une grande proportion de ses lecteurs. Mais si elle en a profité, elle a aussi payé le prix de la conformité aux normes de son temps, dans l'autocensure qui l'a empêchée de terminer les deux romans (Maternité et La lampe est éteinte) qui jaillissaient de sa propre expérience et qui étaient peut-être trop révélateurs de ses angoisses pour qu'elle puisse les offrir au public. Ses œuvres publiées ont la saveur d'un autre temps; ce sont trop souvent des histoires à l'eau de rose, baignant dans une nostalgie du passé ou tournant autour d'épisodes invraisemblables qui trahissent le manque d'inspiration dont elle se plaignait souvent dans son journal. Il se peut que la plus grande contribution de Michelle Le Normand à la littérature soit le journal auquel elle a confié l'essentiel de sa vie pendant plus de cinquante ans, avec ses émotions prises sur le vif, ses interrogations profondes sur le sens de la vie et la passion de l'écriture qui le traverse et qui est au fond sa principale raison d'être; passion secrète et fondamentale à son existence, que Le Normand décrit avec éloquence dans un texte de 1941 intitulé «Délice, tourment d'écrire»:

18 Suite romanesque de huit volumes (Paris, Librairie Gallimard, 1922-1940). 19 André Gide, L'immoraliste, Paris, Mercure de France, 1902, 259 p. 20 Henry de Montherlant, Les jeunes filles, Paris, Grasset, 4 vol., 19361939. 21 Georges Bernanos, Journal d'un curé de campagne, Paris, Plon, coll. «La Palatine», 1936, 366 p. 22 François Mauriac, La pharisienne, Paris, Grasset, 1941, 296 p. 
Tous croient que vous n'écrivez que pour vous amuser [...]. Et personne ne sait la vérité, personne ne l'imagine, personne ne comprend que, pour vous, écrire est la question primordiale, celle qui règle la joie de vos jours, celle qui, à tout instant, tinte en vous - comme un reproche, si les soirs achèvent des journées vides, - comme un joyeux son de cloche, si les dernières pages noircies sont bonnes, nombreuses... Plutôt que de le sacrifier, vous refuseriez tout. Car pour vous rien ne vaut ce tourment, ce délice d'écrire, qui vous gâte parfois les plaisirs ordinaires du monde, mais qui vous remplit plus souvent d'une joie unique [...].

Délice de créer, de voir pousser les phrases comme des fleurs dans un magique jardin, de les voir pousser et à votre insu se ranger, s'agencer, compléter votre idée, l'éclairer d'une lumière que tout d'abord vous n'aviez pas soupçonnée...

Délice, tourment d'écrire. Bien qui console des douleurs, du temps qui passe, des pauvretés, des injustices, bien qui console de tout ${ }^{23}$.

23 Michelle Le Normand, La maison aux phlox, p. 7-9. 